\title{
Xanthogranulomatous Cholecystitis
}

National Cancer Institute

\section{Source}

National Cancer Institute. Xanthogranulomatous Cholecystitis. NCI Thesaurus. Code C35792.

Cholecystitis that is characterized by nodules containing lipid. 